(NASA-CR-199788) THREE-DIMENSIONAL

N96-15638 AERODYNAMIC SHAPE OPTIMIZATION

USING OISCRETE SENSITIVITY ANALYSIS

(OId Dominion Univ.) 2 p

Unclas

$63 / 05 \quad 0083098$ 
ABSTRACT

\section{THREE-DIMENSIONAL AERODYNAMIC SHAPE OPTIMIZATION USING DISCRETE SENSITIVITY ANALYSIS}

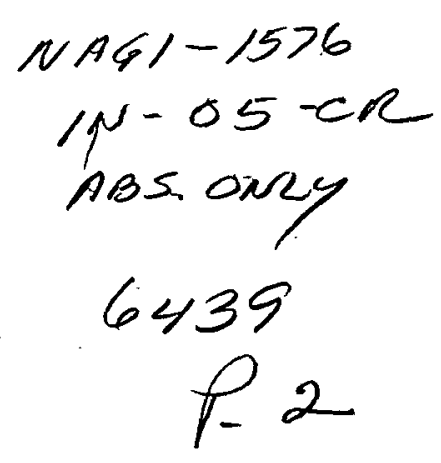

\author{
Gregory W. Burgreen \\ Old Dominion University \\ Director: Dr. O. Baysal
}

An aerodynamic shape optimization procedure based on discrete sensitivity analysis is extended to treat three-dimensional geometries. The function of sensitivity analysis is to directly couple computational fluid dynamics (CFD) with numerical optimization techniques, which facilitutes the construction of efficient direct-design methods. The development of a practical three-dimensional design procedures entails many challenges, such as: 1) the demand for significant efficiency improvements over current design methods; 2) a general and flexible three-dimensional surface representation; and 3) the efficient solution of very large systems of linear algebraic equations. It is demonstrated that each of these challenges is overcome by: 1) einploying fully implicit (Newton) methods for the CFD analyses; 2) adopting a Bezier-Bemstein polynomial parameterization of two- and three-dimensional surfaces; and 3) using preconditioned conjugate gradient-like linear system solvers. Whereas each of these extensions independently yields an improvement in computational efficiency, the combined effect of implementing all the extensions simultaneously results in a significant factor of 50 decrease in computational time and a factor of eight reduction in memory over the most efticient design strategies in current use. The new aerodynamic shape optimization procedure is demonstrated in the design of both two- and three-dimensional inviscid aerodynamic problems including a two-dimensional 
sippersonic internal/external nozzle, two-dimensional transonic airfoils (resulting in supercritical shapes), three-dimensional transonic transport wings, and three-dimensional supersonic delta wings. Each design application results in realistic and useful optimized shapes. 